\title{
STUDY ON WEB-BASED AGRICULTURAL MECHANIZATION DECISION SUPPORT SYSTEM
}

Minli Yang, Feng Yang, Lijuan Shi, Jieqiang Zhuo

College of Engineering, China Agriculture University, No. 17 Qinghua Donglu, Haidian District, Beijing, China

Abstract: The paper discussed the background and meaning of research on Agricultural Mechanization Decision Support System (AMDSS). The exploitation idea of AMDSS based on web was brought forward. The data mining technique was put into the system in order to make its functions stronger and realization easier. Also designs were proposed on general framework and modules for AMDSS.

Key words: Agricultural Mechanization, DSS, Web, Data Mining

\section{FOREWORD}

Decision Support System (DSS) is the newest developing stage in the information system research. It is a decision-supported knowledge and information system, which bases on the computer technology and is used for dealing with the semi-structured and unstructured problems during the decision process. The research of DSS started at the beginning of the seventies of the 20th century. After more than 30 years of development, DSS and it's relevant technologies have been regarded as the accessorial support tool of administrative decision, widely used in such aspects as agriculture, industry, commerce etc., and become the important research subject in the system engineering and computer field. ${ }^{[1]}$ The rapid development of the computer technology plays a strong role to DSS technology. The fast popularization of WWW technology, the mode of calculation taking network as the centre becomes the mainstream, the combination of various kinds of 
advanced communication technology and DSS is the inevitable trend of DSS development.

The agricultural mechanization system is a complicated system based on the regional natural resources and the social economic condition, and its macroscopical decision is a complicated course. The agricultural mechanization department as the policymaker should fully consider the social economic and natural conditions in a certain area. Also it should utilize the high-quality information effectively and in time in order to serve for adjusting the market and macroscopical decision of rural economy. So, it is urgent to establish an open Agricultural Mechanization Decision Support System (AMDSS), which regards aid decision as object. This AMDSS can offer various kinds of integrated data information, suitable models and analytical methods to support the agricultural administrative staffs to solve various kinds of decision problems or make relevant development plans.

\section{DESIGN FOR THE GENERAL FRAMEWORK}

The problems, which the staffs meet during the agricultural mechanization decision-making process, belong to semi-structured and unstructured problems. So-called structured degree means that the environment and law of a certain process could be offered clear explanation or description by the specific language (mathematics or logic, formal or informal, quantitative or rational). If they can be described clearly, they are called the structured problems; if they can't describe clearly, and only can be judged by intuition or experience, they are called unstructured problems; other problems lie between the two, are called semi-structured problems. ${ }^{[2]}$

As AMDSS involves an amount of the data and economic information of agricultural mechanization, and involve a wide range, including the area, the affiliated province and whole China, and according to the latest direction of DSS development at present, the Data Warehouse, the On Line Analytical Process and Data Mining technology were adopted into AMDSS to form a comprehensive DSS. Also the information of agricultural mechanization information net and economic information used in AMDSS to make the whole AMDSS connect with INTERNET. AMDSS is divided into 3 modules: Data Warehouse Management System, Data Mining System and Model Management System. AMDSS structure is as Fig.1. 


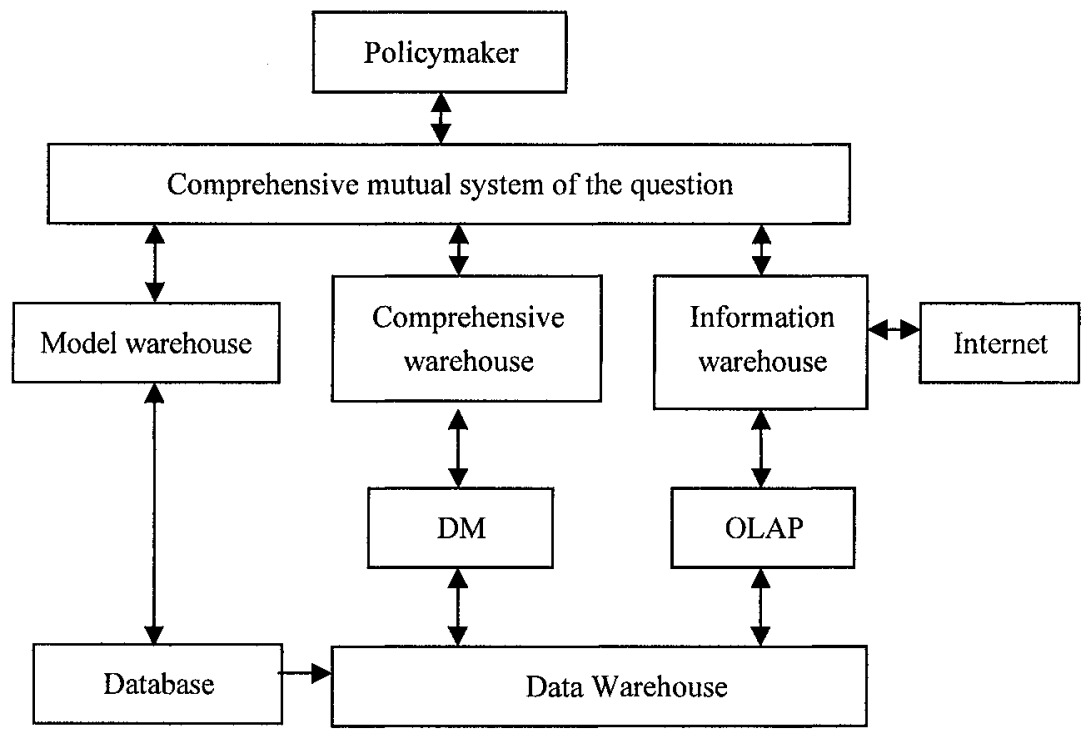

Figurel AMDSS structure on the basis of the data warehouse

\section{CHOOSING AMDSS DEVELOPING PLATFORM}

There must be a good developing tool to popularize the DSS. It requires a language system to combine the data module, model module and dialogue module together. Data module and model module has separate riper language systems each other. The traditional algorithmic language such as FORTRAN, PASCAL, etc., suits the realization of the mathematics model very much, but does not support the operation of the database.$^{[3]}$ Various kinds of DBMS language are suitable for the management and operation of the database, but not suitable for number value operation. Integrated language should synthesize the calculate language and the database language. Statistical analysis system (SAS) is the most famous data analysis software system nowadays in the world. In latter sixties of the 20th century, SAS was developed by Prof. A. J. Barr and Prof. J. H. Goodnight in U.S.A. North Carolina State University. They established U.S.A.SAS research institute in 1975, and then put out SAS system. SAS was world-famous for leading technology and reliable support all the time. Through the constant development and improving of more than 30 years, SAS have already become the large-scale integrated application software system at present. After 1990s, SAS was devoted to develop the data warehouse, the on-lined 
analyze and process system, data mining and DSS. And now, the more successful large-scale software SAS8.0 integrates calculating language with database language to be simpler and more direct than other language such as FORTRAN etc. in calculating in number value. ${ }^{[4]}$ So, we choose SAS8.2 as the platform to develop the whole system.

\section{DESIGN OF EVERY MODULE}

\subsection{Setting-up the Data Warehouse System}

The data warehouse is a theme-oriented, integrated and steady data collection in different time, which is used to support the decision formulation process in management. The data in warehouse faces theme correspond to traditional database face applying. Theme is a standard which classify the data in higher level, and every theme corresponds to one macroscopic analysis field. The integrated characteristic of the data warehouse refers to the process that before entering the data warehouse, the data must be processed and integrated. It is the key step of setting up data warehouse to unify the contradiction of the initial data and to make the data structure a change from face applying to face theme. The stability of the data warehouse means the data warehouse reflects the content of the historical data, but not the data produces in daily transaction processing, the data are seldom revised or not revised at all after processing and integrating and entering the data warehouse. Data warehouse is the collection of data in different time. It requires the data saved in warehouse to keep enough time in order to meet the requirement to carry on decision analyses. The data in warehouse should show its historical periods. ${ }^{[5]}$

Meta-data is another important concept in the data warehouse. It is usually defined as data that is related with others. Meta-data refers to data that describes other objects. These descriptions are used to define tabulation, database, view and other objects in relational database. Generally, Meta-data represents anything that is used to define the objects of data warehouse, and the objects may be tabulation, a list, a query, a commerce rule or metastasis in the data warehouse. The definitions of meta-data help us finish the definition of the entire significative object in the data.

The purpose of studying AMDSS is solving management and decision problems during the development of agricultural mechanization. However, the agricultural mechanization system is an open system, which is influenced 
by the external population, resource, environment, economic development, agricultural production, technological progress and market demand, etc.. In order to meet the research requirement, the data warehouse should embody the data of agricultural production, industrial structure, agricultural workforce proportion, agricultural output value etc. in every region in China and all over the country. The researching content is mainly to solve the problems of agricultural mechanization development management and decision. So, the setting-up of the data warehouse is mainly around these respects. Its themes are chosen according to the content of the data but not the difference of the area. The same content data is belonged to the same theme. The same data in different areas is gathered into the collection, and then compared and analyzed. Processed data is put into the information market. Also it sets up several data markets in the warehouse in order to meet the demands of the different levels' managers for data.

\subsection{The Development of Data Mining System}

Data Mining (DM) is getting the knowledge that people are interested in from the large-scale database. The knowledge is connotative, potential useful information which is not known in advance. And the drawn knowledge is expressed as concept, rule, law, mode, etc.; it means looking for the mode of decision support process in the aggregation of facts and data. ${ }^{[6]}$ The target that the data mining is not only the database, but also file system, or other data aggregations, such as WWW information resources.

All the research of data mining is base on the data warehouse which we have set up, and it form a structure with data warehouse, manager and decision tools as Fig. 2. It has the following functions: (1) Simple- data sampling; (2) Explore - exploring, analyzing and pre-treating the characteristics of the data; (3) Modify-defining the problems, adjusting the data and choosing the technology; (4) Model-researching the model and discovery the knowledge; (5) Assess - comprehensive explaining and appraising of the model and knowledge.

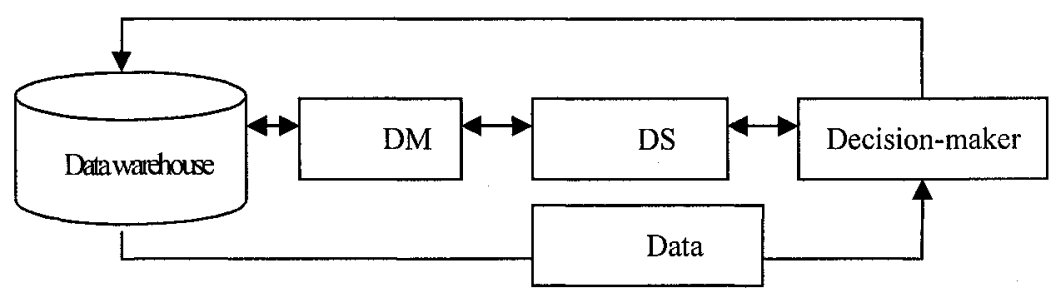

Figure 2 Data Mining under data warehouse environment 


\subsection{The Model Warehouse and Managment System}

The model is the description of the essential characteristics of a system in a certain form to describe the function, behavior and the rules of change of the system. Generally, we have 3 kinds of methods, including logical oreganos method, experiments method and models method, to understand and research the objective world. The model method is the most capable, convenient and effective way to understand and explore the objective world. And it erects a bridge between the objective world and the scientific theory to analyze all aspects of the system. The multiformity and complexity of agricultural mechanization bring a lot of difficulties to research its development. The foundation and analyses of model is helpful to its development. And the operation of the model is help to optimize its structure.

The model warehouse is the computer system to offer storage and express, which is named "the model management system". There is over one proper memory mode to acquire, visit, refresh and synthesize the model in it. In this system, the first thing we should think over is take a propel express and storage way in order to manage the model, connect in a flexible way and participate illation. The express way of model tends to disassemble to the basic cell so as to strengthen management flexibility and reduce the storage redundancy. AMDSS adopts data mining technology under SAS platform. So, the model can realize in the data mining in general DSS. This reduces the model quantities in the storehouse and developing difficulties of management system and the whole system.

The OR model in SAS system includes the common planning method. This kind of question can be considered as a problem of solving the maximum of a goal function under a group of restrictions. If the restrictions and goal functions are linear function, it is the Linear Programming (LP). If the variable must be the integer, it is the Integer Plan (IP). Process LP in the OR model can be solved by the LP, which includes the integer plan and mixed integer plan. Generally, planning that are used in AMDSS is linear programming. The restraint terms, parameters and goal function of the LP can be stored in the database, and some of parameters can directly get from the database. Operation only need to transfer LP program and input the data from the database. When the model changes, it can finish the operation and management at the same time through the parameters in the data file only are revised. It simplifies the workflow of the model management system, and improves working efficiency. As follow Fig. 3. 


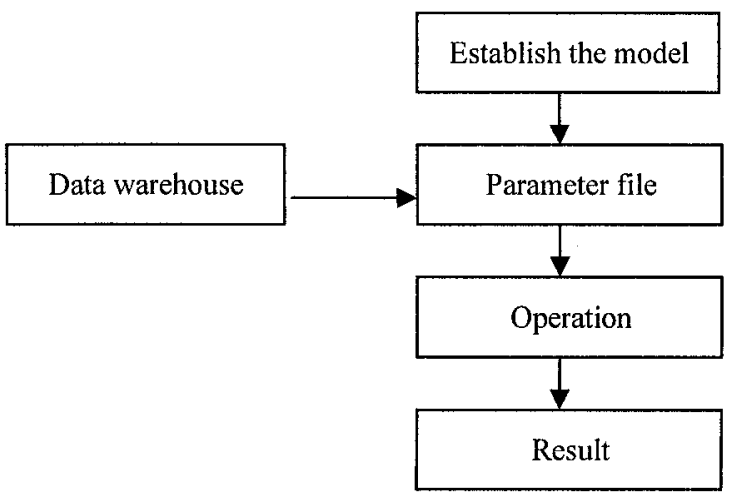

Figure 3 The development of the model administrative system on the basis of SAS system

\section{INTEGRATING OF AMDSS SOFTWARE SYSTEM}

Because the data warehouse system, data mining system and model administrative system of AMDSS are developed under the systematic platform in SAS, and the source of the data mining is the data warehouse. So, the data mining and the establishment of data warehouse link seamlessly. (Fig. 4) In the model management system, the process of AMDSS developing is: (1) obtaining the data from the data warehouse through the data file of the model; (2) finishing the development of every module and the module interface in the data warehouse. Integrating research results is as Fig. 5 .

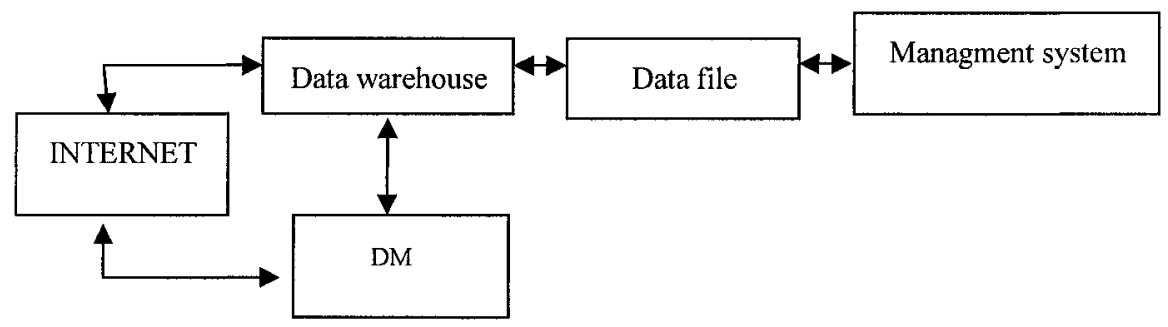

Figure 4 Connection of the module in AMDSS 


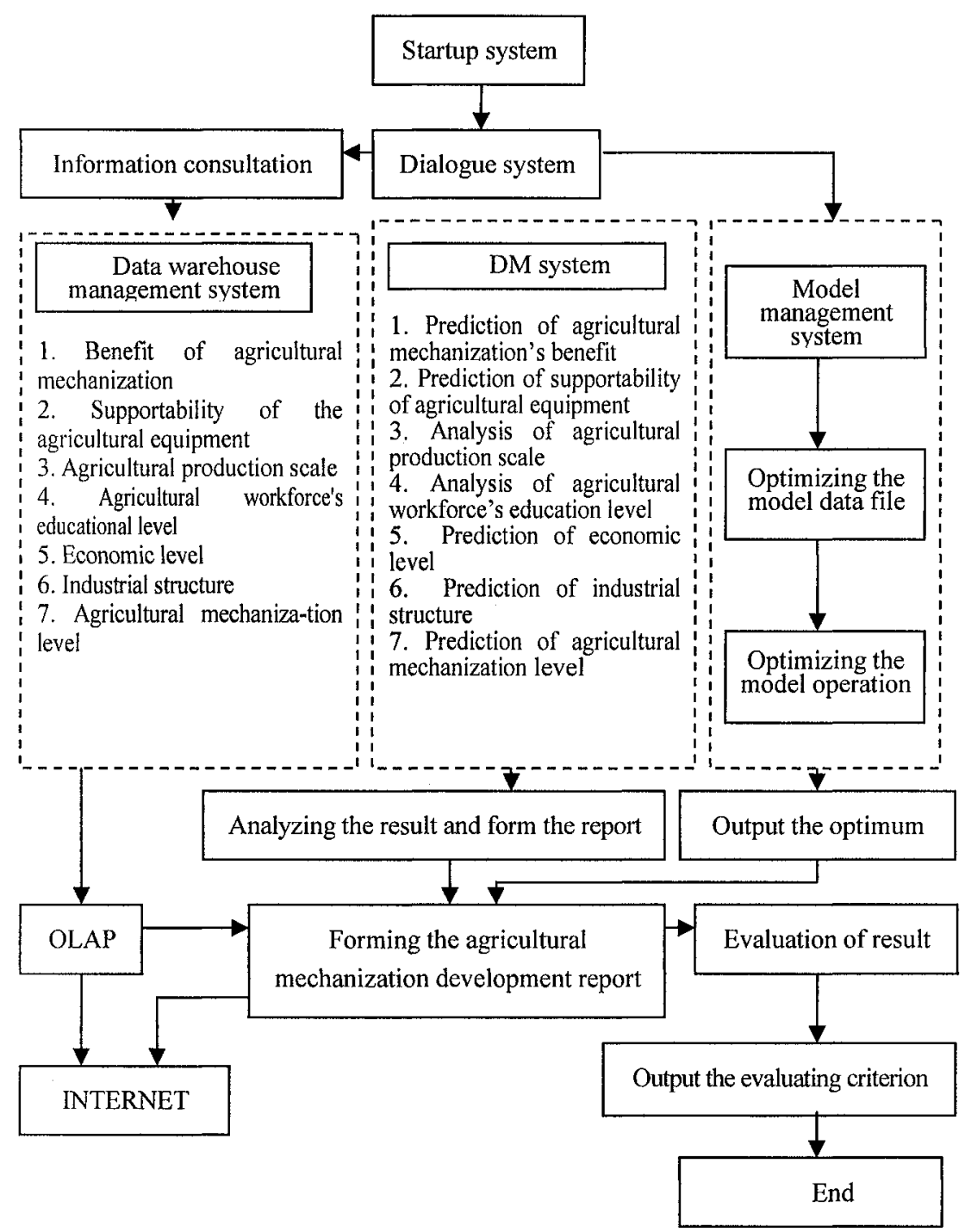

Figure 5 AMDSS operation structure

\section{CONCLUSIONS}

AMDSS has used in China every province and all over the country to appraisal and make policy of the agricultural mechanization development. Some decisions have been implemented. Agricultural information is one of the developing directions of future agriculture, and AMDSS is an important 
part of it. AMDSS is sure to play more and more important role in the agriculture development in future.

\section{REFERENCES}

[1] Liu Yao. On Application and Development of the Decision Support System. Computer and Modernization, 2000(2): 41 47

[2] Meng Ju, Yang Guanglin. The Research of Agricultural Producing Macro Decision Support System. System Sciences and Comprehensive Studies in Agriculture, 2002, 18(4): 293 297

[3] Cheng Weiwen. Decision Support System and its Development. Beijing: Tshinghua University Press, 2000

[4] Gao Hongshen. Theories, Methods and Cases for DSS. Beijing: Tshinghua University Press, 2000

[5] Weston J., author; Chen Shuoying, etc., translater. Decision Support in the Data Warehouse. Beijing: Beijing Institute of Technology Press, 2000

[6] Jiawei Han, Micheline Kamber, author; Fang Ming, Meng Xiaofeng, translater. The Concept and Technology of Data Mining. Beijing: China Machine Press, 2001 